\title{
Transcending adversity: resilience in volunteer firefighters
}

\author{
Leigh McCarley Blaney \\ Bachelor of Science in Nursing, Vancouver Island University, Nanaimo, Canada \\ David Wilde \\ Nottingham Trent University, Nottingham, UK, and \\ Rowena Hill \\ Psychology, Nottingham Trent University, Nottingham, UK
}

Received 31 October 2019

Revised 1 June 2020

11 September 2020

10 November 2020

Accepted 20 November 2020

\begin{abstract}
Purpose: The purpose of this paper is to present a theory of psychological resilience in volunteer firefighters.

Design: Using a constructivist grounded theory (CGT) approach, the qualitative study engaged a purposive sample of eight firefighters in Canada, conducted in-depth interviews, and analyzed the data using comparative methods.

Findings: The results provided unique insights into resilience in firefighters, and revealing resilience as multidimensional, complex, dynamic, and contextual. Six core concepts inter-relate to construct resilience: relationships, personal resources, meaning-making, leadership, culture, and knowledge.

Practical implications: The findings of this research offer a framework for practical integration of resilience theory into workplace health policy and practice. The theory was co-created with firefighters hence is contextually sound to this population but applicable to other emergency and health services.

Originality/value: Volunteer firefighters are under-represented in the literature despite facing intermittent and frequently intense work-related stressors; this research begins to address the gap in literature. As well, previous resilience theories have noted relationships between some components, but there is little evidence linking categories; this theory more patently represents the complex nature of resilience in volunteer firefighters.
\end{abstract}


Keywords: resilience, volunteer firefighters, constructivist grounded theory, workplace health Introduction:

The intent of this article is to discuss a Constructivist Grounded Theory (CGT) study that was designed to explore and theorize how volunteer firefighters understand and operationalize the concept of resilience.

The phenomenon of resilience has been posited as a contributing factor to mental health, in individuals and communities, following adversity (Southwick et al., 2011), and resilience has been found to be common following adversity (see for example: Bonanno, 2004; Masten, 2001). In a study with volunteer and career firefighters (Blaney \& Brunsden, 2015), firefighters scored moderately high on the Resilience Scale (Wagnild \& Young, 1993); however, the interpretation, meaning, and understanding of resilience in the fire rescue service (FRS) has not been explored leaving gaps in the resilience literature and questions about how and why firefighters are resilient.

From a workplace health perspective, the FRS is considered to be a 'high risk' occupation, one that carries significant risk of physical and psychological sequelae to the job (British Columbia Firefighters Association, 1999; Haynes \& Molis, 2015). The literature on firefighter mental health is dated and has primarily focused on 'illness' outcomes such as posttraumatic stress disorder (PTSD) (see for example: Bryant \& Harvey, 1995; Haslam \& Mallon, 2003). However, firefighters have a reasonably low incidence of PTSD relative to other emergency service providers such as police and paramedics (see for example: Alexander, 2015;

\footnotetext{
We thank the firefighters who participated in this research; your contributions to our knowledge of resilience are immeasurable. We appreciate each firefighter who, with courage, sacrifice, and humility, serves our communities; may our increasing knowledge of resilience buoy you throughout your career.
} 
del Ben et al., 2006; Halpern et al., 2009); there is little recent research related to the how and why of these low rates, nor into the role of resilience in adult, non-clinical populations such as firefighters (Blaney \& Brunsden, 2015; de Terte et al., 2014).

It is important to understand resilience from the perspective of volunteer firefighters since volunteers make up the majority (over 85\%) of firefighters worldwide (Karter \& Stein, 2013; National Fire Protection Association, 2016). The term 'volunteer' in this research refers to those firefighters who are not making a career of firefighting and who generally receive little financial compensation for their firefighter work.

There is limited literature about volunteer firefighters yet assumptions are made that research on career firefighters and other emergency service providers applies to volunteers (see for example: Beaton et al., 1998; Wagner et al., 2010); this may well be the case but currently there is little evidence to support this assumption. Also, much of the stress literature with firefighters is dated although there is a developing body of evidence that contrasts with the older literature in relation to how firefighters perceive and cope with stressors in the FRS (see for example: Jeannette \& Scorboria, 2008; Johnson, 2010). As well there is developing evidence of differences in how volunteer firefighters and career firefighters understand and interpret stressors, what incidents constitute 'traumatic events' and what strategies are commonly used to cope with post-event distress (Blaney \& Brunsden, 2015; Johnson, 2010). It is important to explicate firefighters' definitions of resilience because it is a term that is used frequently in the FRS, and in occupational health services (Mental Health Commission of Canada, 2013), and resilience in volunteer firefighters may or may not align with existing understandings of the concept. 
Interpretation and understanding of resilience has evolved through the work of many researchers although the majority of resilience research has occurred with clinical populations (Masten, 2001; Richardson, 2002; Ungar, 2011). Resilience has been explored with only a few non-clinical or 'healthy' populations (see for example: Bonanno, Brewin, et al., 2010; Wagnild \& Young, 1993) which means that understanding of resilience may be skewed by a deficits or illness perspective. Mancini and Bonanno (2009) assert that illness cannot be effectively understood or managed without a clear and deep understanding of resilience and health; this view is in keeping with a 'health promotion' perspective of resilience that focuses on strengths and capacities (Antonovsky, 1996; Blaney \& Brunsden, 2015) and runs counter to the typical exploration of resilience from a reductionist deficits perspective (see for example: Richardson, 2002). However, the current research aimed to counter the hegemony of conceptualizing resilience as a linear corollary of illness.

Although various contexts are considered in the resilience literature, the majority focus on developmental resilience to long-term adversity (see for example: Masten \& Narayan, 2012; Ungar, 2011). Relevant to firefighters who are assessed as being at high-risk of workplace psychological injury is the significant gap in the literature exploring alternative outcomes to injury, such as resilience. In addition, the population and context of firefighters is quite different from the developmental literature in that they are adults who generally lead healthy lives physically and mentally, yet are routinely exposed to intense short-term work-related adversity (Jeannette \& Scorboria, 2008; Johnson, 2010); the majority of firefighters are very successful in their adaptation to stressors (del Ben et al., 2006).

Multiple factors are implicated in resilience: flexibility in access to and use of resources or assets (Hobfall, 2014), workplace health promotion (Noblet \& LaMontagne, 2006), sense of 
coherence (Antonovsky, 1996), and social context (social support, work culture, etc.) have all been implicated in defining and enhancing resilience (Chopko \& Schwartz, 2009; ShakespeareFinch, 2011; Tehrani, 2011). The evolving understanding of factors that can influence resilience are relevant to volunteer firefighters since there is clear evidence that resilience is a complex contextual and adaptive process (Ellis, 2015) that can be taught and strengthened.

In summary, while research has explored resilience for many years, there are considerable gaps in relation to understanding resilience as more than the absence of psychopathology and in the context of adult non-clinical populations, especially firefighters and, more substantively, volunteer firefighters. Previous work has taken a nomothetic approach, leaving gaps the literature; in contrast, this study takes an idiographic approach and enters the world of volunteer firefighters by asking how they understand and experience resilience.

\section{Aims of the Study}

Two questions relevant to this qualitative study were: "what are the core concepts of resilience in the volunteer FRS?', and 'what are the relationships between and among the core concepts?' The emergent firefighter data in response to these questions was used to construct a theory unique to pre-existing theories of resilience (see for example: de Terte et al., 2014; Richardson, 2002) yet is congruent with the dynamic nature of the construct within this context.

\section{Method}

\section{Study design}

This study explored and theorized a resilience construct in volunteer firefighters; CGT is the methodology of choice when exploring the "how and why things happen" (Mayan, 2009, p. 
35). CGT is essentially the collection, analysis, and making meaning of data; it is systematic, inductive, and uses comparative methods to 'make sense of' (Vann-Ward et al., 2017) and explicate the phenomena of resilience in volunteer firefighters; the participants and the researcher are co-constructors of the theory (Charmaz, 2014). The study did not explicitly seek to control factors or variables, nor did the research team use or impose a specific definition of resilience; the study was designed to examine and explicate the core concepts of firefighter resilience and the relationships between/among those concepts.

\section{Sampling and participants}

Setting

The study was set in Canada primarily because the principal investigator lives and works in Canada and has access to a large pool of volunteer FRS. In order to preserve anonymity of participants, the pseudonym Anonymous FRS (AFRS) was assigned to the FRS under study; AFRS is typical of many volunteer FRS in its governance, demographics of community and FRS membership, geography, and its 'service orientation' and close relationship to the community. It was invaluable to this study to work with an FRS that is prototypical in order to lay the foundation for resonance, credibility, and usefulness of the constructed grounded theory, three of the four criteria for evaluating grounded theory studies (Charmaz, 2014). Other commonalities across volunteer FRS in Canada include for example: firefighters are trained as medical first responders which adds a layer of interaction with human suffering that is not as present in FRS who may not be medically trained (such as FRS in Europe); as well volunteer firefighters are also routinely responding to distressed people who may be their family, friends, or neighbours. 
In contrast to its typicality, AFRS is innovative in its proactive orientation towards health and health promotion health literacy (i.e. psychological health initiatives such as a Critical Incident Stress Management (CISM) program); familiarization with health constructs and increased knowledge about reactions and coping with stressors have been linked to resilience (Southwick et al., 2014). The health orientation and non-clinical population of this study contributes originality to the exploration of resilience in volunteer firefighters.

Sampling was purposive and taken from FRS that met inclusion criteria for study such as: the FRS is a volunteer department; project participation was available to all members of the FRS regardless of rank or role; the FRS serves urban and rural geographical area. .

\section{Participants}

There were ten firefighters from AFRS (about 1/3 of the active members in the department) and two external participants or advisors (EAs). The EAs were treated as research participants vis-à-vis the consent and interview processes. The role of the EAs was to both challenge and support the evolving grounded theory; they were conveniently selected to participate - one was a firefighter from a different geographical area and one was a psychologist with experience in working with firefighter clinical populations and who had been trained/oriented to a volunteer FRS. Ultimately two AFRS firefighters were lost after the first interview - one withdrew due to geographical relocation and one died; their data is excluded. The remaining eight firefighters (about $1 / 4$ of the FRS membership) and the advisors contributed over 50 hours of interview data. Participant demographics are described in aggregate in order to maintain anonymity, with each participant being randomly assigned a gender-neutral pseudonym for purposes of tracking during data collection and analysis. The demographics are summarized in Table 1 below: 
Table 1: Demographics: $n=8$

\begin{tabular}{|l|l|}
\hline Gender & $\begin{array}{l}\text { Self-identified as male 7 } \\
\text { Self-identified as female 1 }\end{array}$ \\
\hline Age & $\begin{array}{l}\text { Early 20s to mid-60s } \\
\text { Mean age 48 years }\end{array}$ \\
\hline Years of service & $\begin{array}{l}\text { 4-30 years } \\
\text { Mean years of service 20 years }\end{array}$ \\
\hline $\begin{array}{l}\text { Married or in committed } \\
\text { personal relationship }\end{array}$ & $\begin{array}{l}\text { In committed relationships } 7 \\
\text { Identified as 'single' 1 }\end{array}$ \\
\hline $\begin{array}{l}\text { Engaged in full-time work } \\
\text { outside the volunteer FRS }\end{array}$ & \\
\hline $\begin{array}{l}\text { Retired from full-time work } \\
\text { but take on extra tasks at } \\
\text { the FRS }\end{array}$ & \\
\hline $\begin{array}{l}\text { Involved in volunteer work } \\
\text { in addition to the FRS }\end{array}$ & \\
\hline $\begin{array}{l}\text { Live within the geographic } \\
\text { confines of the FRS hence } \\
\text { are in contact with } \\
\text { community members during } \\
\text { emergency calls as well as } \\
\text { when off-duty from FRS }\end{array}$ & \multicolumn{1}{|c|}{$\mathbf{2}$} \\
\hline
\end{tabular}

As well, once the theory had been constructed, scrutinized and critiqued by the research team and participants, a convenience sample of three career firefighters and two nurses was recruited. The role of this sample was to evaluate the emerging theory against criteria for CGT: originality, credibility, resonance, and usefulness (Charmaz, 2014) across a variety of contexts; again, all were bound by the same ethical guidelines as the original participants and EAs.

\section{Data collection and analysis}


The study received favourable ethical approval by the host institution's Research Ethics Board.

Three face-to-face interviews were carried out with each of the eight AFRS participants and two EAs resulting in about 50 hours of rich robust data. Interviews were conducted following a flexible, evolutional interview guide comprising open-ended questions and prompts (i.e. "Tell me a story/give me an example about you and resilience in your fire career - about a timeltimes when you were resilient"; "What are the markers of resilience in/for you?"). The interviews ranged in duration from 60-90 minutes, and were digitally recorded and transcribed verbatim prior to analysis.

Data analysis adhered to the guidelines of Charmaz (2014). Theory construction began with lineby-line analysis of interviews to ensure fidelity with the data. Initial, focused, and theoretical coding followed which allowed the categories, and the relationships between categories, to emerge. In order to ensure fit with the data, details of the categories and the relationships were examined; memos provided the 'map' toward theory construction by exploring relationships and tracking ideas, and further conceptualizing categories. Constant comparison and negative case analysis were utilized to establish analytic distinctions and to search for cases that refuted the categories. Transcripts were not presented to participants but as the theory was emerging concurrent interviews allowed for scrutiny of categories and connections, and for clarification with participants. The theory was co-constructed through the back-and-forth integrative process between the researchers, the data, and the participants. Once constructed the CGT went back to the participants for critique and confirmation, and was presented in a draft form to firefighters in the third interview where interview questions were generally specific to the theory overall.

\section{Results}


In direct relation to the first research question ("what are the core concepts of resilience in volunteer firefighters') the data analysis surfaced several categories, which were distilled or consolidated with further interviews and analysis. Ultimately, six categories: relationships, personal resources, meaning-making, culture, leadership, and knowledge were seen to be 'core' categories, and had several sub-categories attached to them and to each other. It is important to remember however that the core categories of resilience do not exist in isolation of one another; instead, they are reciprocally inter-related and reflect the "social processes" (Charmaz, 2014, p. 35) of volunteer firefighters hence addressing the second research question ('what are the relationships between and among the core concepts'). The six categories together reflect a 'systems' (von Bertalanffy, 1969) approach to defining resilience (the emergent substantive theory is summarised in Figure 1).

\section{[Insert Figure 1 here]}

\section{Discussion}

\section{Core categories of resilience}

The following sections descriptively define and discuss the six core categories of the emergent theory (Blaney, 2017). Excerpts from interviews are included as examples however have been reduced to short selections in order to illustrate the core categories; they do not fully represent the depth and breadth of firefighter perspectives. For this article participants were given alphanumeric codes (i.e. Firefighter 1 aka FF1) to differentiate the source of the excerpts. Relationships 
Often named 'social support' in the literature (de Terte, et al., 2014; Fletcher \& Sarkar, 2012), this category emerged from the data as 'relationships'. It is evident that social support is an accurate description of the process that occurs between firefighters and others; however that support is underpinned by relationships that are constantly negotiated in order to optimize social support. The category of relationships includes firefighters' chosen coping strategy of 'talking about' the impact incidents have on them and how they will cope with those. The people that firefighters speak to most frequently are their fellow firefighters, closely followed by their spouse and significant others.

Familial relationships were routinely named as is a significant cornerstone to resilience: [If the call has] been stressful or something there's always ... a sandwich ...cup of tea...something to take the edge off... and the offer of a listening ear...[My spouse] will know because they have access to radio or pager and listen to the call... (FF3)

The importance of negotiation with spouse/family about time spent with the FRS, both on call and also involved in community events such as fundraising, attending parades, etc. requires ongoing commitment and communication:

It's a delicate balancing act - it always is, no matter how long you do this work - it's constant negotiation between the demands here [the FRS] and your family. (FF8)

The literature links effective and ongoing communication to healthy relationships which in turn correlates with resilience (Regehr, 2009). Knowledge of the role the family plays in sustaining and enhancing resilience in firefighters behoves us to pay close attention to also building resilience in firefighter families. McMahon (2010) noted that support from family and friends requires negotiation, effective communication, and education of family members as to the stressors encountered in the FRS, a view that is echoed by participants in this study. Hill (2015) 
suggests several ecologically relevant processes to support families including: creation and delivery of reliable credible messages to families focusing on normalizing the job, stressors, and resilience along with family outreach such as invitations to training days; the data in this study highlight families, communication, and negotiation as key components of relationships.

Social support has long been implicated as a key component of resilience and there is much evidence around the association of social support with firefighter resilience (Haslam \& Mallon, 2003; Jeannette \& Scorboria, 2009). In the AFRS, social support is not a random experience but is embedded in the relationships of the firefighters - primarily families and colleagues or 'pseudo-family' within the FRS. As underscored by the data, these relationships require nurturing and negotiation in order to remain paramount despite the time and energy required by expectations and tasks within the FRS.

In summary, the data emphasizes several key features of relationships: relationships means more than social support, and the broader understanding augments the existing literature on resilienc; peers and families are the most common sources of relationship, and 'talking' is said to be one of the most helpful sub-categories of relationships as well as of personal resources and culture. Also the context of the volunteer FRS requires specific efforts to nurture family relationships given the multiple demands on the firefighter, and the family, in addition to their role as firefighter. Firefighters at AFRS count relationships as a mainstay of resilience, and in keeping with the literature find social networks offer emotional support, information, and provide tangible and intangible assistance.

\section{Personal Resources}

The category of personal resources has been synonymous with the term 'coping', however, personal resources are the assets and coping is the action; coping cannot occur without 
resources (Hobfall et al., 2014). When discussing the personal resources required for resilience in volunteer firefighters, two sub-categories emerged from the data: physical or external resources, and psychological or internal resources; these are the assets available to firefighters that contribute to resilience.

External resources are described by participants as those tools that one employs to 'blow off' stress and to enhance resilience, and include: exercise, meditation, yoga, and various 'activities' that also serve as distraction from the stress reactions. Personal resources in this study also refers to the availability of more innate or internal mechanisms: attitudes (humour, hope, optimism, positivity), and values (service, commitment, integrity), along with integrating/bringing forward 'experience'; these resources may be innate to some firefighters but also can be learned and developed through training and experience in the FRS.

'Exercise' as a personal resource for participants is an intentional and concrete approach to dealing with work-related stressors. Exercise has been linked to resilience (Childs \& de Wit, 2014) and it is a firmly entrenched resource with volunteer firefighters.

As well, participants describe internal resources that are accessed during times of stress and following adversity including emotional, attitudinal, and values-based resources such as hope, humour, etc.

I think 'hopeful' and 'optimistic' pretty much describe how I get through the tough times. (FF8)

In this study a culture of emotional openness allows for 'venting' of reactions in order to move on and/or look for solutions to distress, to decrease stigma associated with emotional responses to stressful incidents, and to recognize the opportunities for being supportive to one another. 
Humour was consistently endorsed by participants as a reliable personal resource such as having a sense of humour and appreciating others' humour. Humour was articulated as both external ("black humour" such as morbid or ironic humour, or "joking around afterwards") and internal ("having a sense of humour"). Humour is used frequently but judiciously as a personal and as a collective resource. Mancini and Bonanno (2009) recognize positive emotions, including humour are key to adaptation to adversity. Sliter et al. (2013) found that use of humour by firefighters affected levels of PTSD and burnout; humour may buffer the emotional and cognitive reactions to traumatic events (Alvarez, 2013; Haslam \& Mallon, 2003). It is important to recognize that humour is not a universal practice and can be misinterpreted, however the evidence supporting the use of humour in the AFRS outweighs the criticisms, and the use of humour was seen as a helpful personal and cultural resource for participants in this study. 'Experience' was also seen by participants as a resource for building resilience - not to the point of being hardened, but recognizing that bad things happen yet firefighters survive those events; this appraisal strategy can also be shared with others:

Seeing it time and time again...[I recognize] I've done the best I can [and] I'll get through it... [and] I can be a role model for the newer members.(FF4)

Personal resources for these firefighters are constructs that aid in appraisal of events as stressful (or not), and those resources can further be called upon following appraisal as assets for meeting adversity, hence co-contribute to resilience. The raw data demonstrated an abundance of positive language and healthy resources; positive attitudes, thinking, and actions are associated with resilience (Mancini \& Bonanno, 2007), and there is a surfeit of these concepts in AFRS. Having a variety of resources to choose from, or having a number of 'tools in the 
toolbox', was recognized as an asset by firefighters, and they articulated a diverse repertoire to choose from.

In summary, the data supports personal resources as a category that goes beyond simply 'coping' with adversity to building a stronger 'core' of resilience; personal resources impact appraisal of events as stressful or not and impact accessing resources to manage stress. Ensuring firefighters have a variety of core resources, the capacity to know when to access those resources, and the flexibility to recognize which one(s) will serve best in a given context are also significant factors in resilience literature (Bonanno, et al., 2011) as well as within the AFRS.

\section{Meaning-making}

Meaning-making is the process of coming to terms with or making sense of events and outcomes; meaning-making differs from 'meaning made' which is the outcome of the meaningmaking process. Meaning-making is a process embedded in resilience literature (see for example: Bonanno \& Dimich, 2013). In the context of volunteer firefighters in this study, there are common themes of firefighters finding meaning in 'doing the best I can'; providing comfort and support are contrasted by the need to find tangible reasons for bad outcomes on calls.

Even though I was so sad for those people/that family, I went over the call and know that we did the best we could, we did a good job, it was technically a really good response with a very bad outcome in spite of our best efforts (FF8)

The process of meaning-making occurs in a variety of ways that are internal (selfreflection, cognitive appraisal) and external (talking to colleagues, family; more formal processes such as group stress defusings where questions are raised such as 'what good do you see coming 
out of this tragedy'). As well, making use of personal resources such as 'reflection', and accessing internal mechanisms such as faith and optimism helps with meaning-making.

Hope is a common theme underpinning resilience in the FRS, with hope for positive outcomes and hope to minimize suffering as ways to make meaning even when calls have a negative outcome.

[We provided] comfort on the worst day of someone's life, in the face of tragedy (FF6)

Ho and Lo (2012) relate meaning-making to hope and optimism which in turn are related to personal resources; these findings are in line with overall literature but are unique in this context.

\section{Culture}

The concept of culture as a category of resilience surfaced early and was consistent throughout interviews. This is in keeping with literature that recommends highlighting culture and context when defining resilience and when developing interventions or programmes to enhance resilience (Panter-Brick, 2014). Culture refers to a system of learned values, beliefs, behaviours, and language shared by a group; this definition is congruent with the understandings that emerged from the data in this study.

Culture is the unspoken way you conduct yourself [within the FRS] (FF7)

Subcategories such as trust and flexibility arose consistently when firefighters discussed culture. As well, participants noted that despite the inherent hierarchy within an organization such as the FRS, healthy culture allows flexibility in the organizational structure; flexibility links 
back to meaning-making and relationships, and adds to the understanding of resilience (Bonanno et al., 2011) in volunteer firefighters.

The term 'brotherhood' has been synonymous with FRS culture in literature and popular media (Regehr et al., 2005). However, when asked about the meaning of this term if it came up in interviews, participants in this study invariably corrected the perception by offering:

Not so much brotherhood as family - it's not the old macho suck-it-up buttercup mentality but more about supporting and understanding one another - it's more like family...(FF3).

The idea that culture contributes to resilience is not new particularly when looking at organizational literature (Biron \& Karanika-Murray, 2012). It seems unusual to hear it highlighted as a contributor to resilience in volunteer firefighters; there is no evidence that culture in relation to firefighter resilience has been explored in the literature making it unique to this study. Volunteer firefighters, in keeping with the literature (Youssef \& Luthans, 2007), have no doubt that cultural components such as hope, optimism, and well-being are related to resilience; they are also clear that positive culture change within the FRS such as flexible hierarchy, valuing diverse perspectives, and collaboration contributes to resilience.

\section{Leadership}

Leadership was another unique finding in this resilience study and participants describe two facets of leadership that are key to resilience: individual and organizational. Individual leadership has been best defined by Drucker (2001) as the personal qualities and skill sets that contribute to surviving and thriving in rapidly changing environments; these qualities and skills have been endorsed by volunteer firefighters as key contributors to resilience. Organizational 
leadership has been shown to contribute to resilience (Tehrani, 2011) through similar skills and processes as individual leaders, and in this study formal leadership is described as flexible, trustworthy, and values-based.

Leadership fosters resilience (FF6)

Organizational leadership is seen as a strong component of resilience - without effective leadership, the people and the organization will become less resilient and may falter. However, leadership at the individual level is seen to support the designated or formal leaders that are part of the traditional hierarchy. The formal leaders in the AFRS have specific qualities that allow flexibility and collaboration despite the hierarchy, which in turn leads to a sense of belonging, sense of purpose, and accountability for all members whether designated or informal leaders. Attitude starts at the top but it's also how we interact, how we 'be' the FRS (FF4Common threads in the data are the inclusivity of the concept of leadership - each member of the volunteer FRS has the potential to lead by example, with insight and perseverance, and with diverse approaches - all of which contribute to a solid core of shared values and culture of resilience.

Everyone in this department is a leader by virtue of the what we do...service to our community (FF2)

There is a significant gap in the literature about the relationship between leadership and resilience. The closest connection appears to be in the developmental and ecological literature that suggests strong role models, skilful caregiving and parenting (Masten, 2015), as well as the support systems and interactions between those systems that aid and sustain childhood resilience (Ungar, 2011). 
Firefighter descriptions of resilience as collaborative, interconnected, relational, and adaptive resonates with the literature on complexity leadership (Uhl-Bien \& Marion, 2008). Complexity leadership is interactive and adaptable with emergent change, and is 'generative' (from the ground up), responding to the day-to-day challenges within a changing organization. The findings of this study are congruent with the emerging literature on complexity leadership and the role of leadership in individual and organizational resilience.

In summary leadership as a category of resilience was another unique finding in the research with volunteer firefighters, yet relates to literature on developmental resilience (Masten, 2001) and the social ecology of resilience (Panter-Brick, 2014). There remain however significant gaps in the literature about leadership including complexity leadership and how it is understood in relation to resilience in high-risk professions such as the FRS.

\section{Knowledge}

The category of 'knowledge' is common within the extant literature on resilience: knowledge, as information gained and information shared, is a mediator for distress and contributor to resilience for children in various contexts such as conflict zones (Masten, 2015; Ungar, 2011). However, knowledge has not been fore-fronted in other models or theories about resilience.

Firefighters in this research recognize that learning is ongoing and that increased knowledge contributes to resilience by building confidence, competence, and capacity; all have been associated with resilience (Masten, 2015). Knowledge is a resource, and a dynamic and situated process (Cote \& Nightingale, 2012) within the FRS; for example, firefighters engage individually and collectively to produce complex products such as fire suppression and medical first responder experts, fire safety technicians, and resilient colleagues. 
Learning self-awareness as well as 'other awareness [helps everyone] figure out ways to work together (FF4)

Additionally the idea of developing 'better, more well-rounded' people is consistent in the data - not only to benefit the FRS and the community from the perspective of safety and competence, but from the personal realization that the competencies acquired in the FRS are transferrable across a wide array of personal and professional contexts.

It's ongoing - we're always learning and developing skills and competencies for firefighting, first responder, life...(FF1)

Summarizing this study's data on the relationship between knowledge and resilience: knowledge changes rapidly and conversely becomes quickly outdated hence learning must be ongoing; knowledge is not hierarchal, but is equalized and shared. Knowledge contributes to resilience (McAllister \& McKinnon, 2009; Seligman et al., 2009) and resilience can be learned. Being forearmed is a key feature of resilience and ongoing information is critical to maintaining currency in the changing understanding of health and resilience. Firefighters are adamant that attending to the psychological learning needs of firefighters is as important as other aspects of FRS training; this view is supported in the literature (Cote \& Nightingale, 2012) about knowledge workers. Overall, having and/or acquiring knowledge is seen as an asset to aid not only the work of the FRS but also to enhance personal and organizational resilience; the more knowledge one accrues, the more likely it is that a solution can be found when faced with challenges on the fire rescue scene and also in one's interpersonal experiences. These perspectives are in keeping with common understandings around teaching and learning, 
particularly with adult learners (Benner et al., 2010), and align with literature linking resilience and knowledge (McAllister \& McKinnon, 2009).

\section{Interactions between Core Categories}

The bi-directional lines on Figure 1 represent the interactions and relationships between categories and sub-categories, the 'temporality' (Bonanno, Romero, et al., 2015) of the theory. As can be seen, one category does not exist on its own but the theory is more than the sum of the categories.

Relationships as one component of resilience, interacts with other categories: a 'culture' of mutuality, trust, diversity; personal resources such as verbal ventilation; leadership (formal and informal) that leads through inclusion; and education/learning about peer support, healthy relationships, and family education.

There is overlap between the category of personal resources and the categories of relationships, meaning-making, and knowledge. For example, personal resources links to the category of knowledge as firefighters willingly shared resources with one another.

Meaning-making as a core category adds depth to understanding firefighter resilience by reinforcing the concept of resilience as a system of dynamic inter-related pathways. Methods of making meaning such as talking to colleagues and/or family are linked to 'relationships' as well as culture and leadership.

Culture connects with a variety of other categories. Culture that supports firefighter resilience is one that 'lives' its values of service and support, encourages and mentors others to be self-aware and open to talking to one another, provides opportunities for meaning-making, and encourages individual and organizational leadership and knowledge-sharing. 
Leadership as a category of resilience interacts with other categories such as:

relationships individually and organizationally (offering friendship/support, ensuring there are resources available within the organization to assist following potentially traumatic events); personal resources (supporting teamwork, verbal ventilation, and humour); culture (supporting the values of the organization); and knowledge (ongoing education on health, health promotion, stress reactions and coping).

Knowledge is a common thread in several categories of resilience (personal resources, social support, leadership, and knowledge) as noted throughout this study. The literature also shows knowledge contributes to resilience (Bonanno et al., 2015; Southwick et al., 2014), reinforcing the findings of this study.

Visualising a three-dimensional model of the theory proved challenging and a search for a visual model led to the Meffert's "gearball" (Meffert, n.d.). This six-sided, six-coloured Rubik's-type puzzle has multiple movable parts and successfully represents the dynamic interactive theoretical categories of resilience and the flexibility of the construct for volunteer firefighters, as these two participants both noted:

...other models try to get people to fit into them - I guess it's easier to explain but real people, real experiences know resilience is not linear nor do we easily 'fit' into simple models (FF8).

The parts are important and give us some place to 'land' within this very complex model, but it is the connections that give resilience its strength and dynamism (FF1)

\section{Contributions of the theory}

During the literature review, several theories emerged that helped inform the 
conceptualization of resilience at the time this research was undertaken: resilience is common and 'ordinary' (Masten, 2001) and can be enhanced; resilience is conceptualized beyond psychopathology; and resilience is a salutogenic (Antonovsky, 1996) or health construct. The findings of this study enrich the knowledge of resilience by the alignment with other disciplines and theoretical frames including but not limited to: organisational theory, complexity leadership, positive psychology, and health.

Transdisciplinarity adds depth and breadth to existing models and contexts by ensuring a relevant resilience framework for FRS as well as other occupational health settings. Further, this theory adds some balance to the discourse on resilience, not to exclude workplace risks and disease, but to harness the strengths and capacities of individuals and organizations and to look at positive health outcomes to adversity.

Practically, this CGT serves as a foundation for the development of policy and practice in psychological health in volunteer FRS as well as a model for future research with other FRS and emergency services. Targeted resilience education with this theory as the curriculum foundation is a specific example of its value, The theory currently underpins curriculum in a study that is exploring the effectiveness of resilience education for firefighters; preliminary results show positive changes to resilience scores and behaviour post-education. The theory has also become part of new recruit training and has been interwoven into CISM education for FRS and other organizations. Within mental health sectors, this theory integrates well into recovery and traumainformed practices, and has been incorporated into community nurses' work with marginalized populations. As well, the theory has been utilized by nursing students who are grappling with their own, and with their patients', COVID-anxiety and grief, allowing the building of assets within each category \& contributing to confidence and competence. Looking ahead Once 
applied, the theory and practices will require ongoing evaluation - another area for future research.

Much of the global discourse on workplace health focuses on the creation and maintenance of a healthy, safe workplace (Mental Health Commission of Canada, 2013). This theory of resilience in volunteer firefighters allows one or more of the resilience categories to be 'start points', with an initial focus on the interface of those categories; for example, developing workplace mindfulness programmes to enhance relationships or personal resources (or combinations of one or more categories) in the workplace.

The strengths of this CGT of resilience are in its complexity, dynamism, and flexibility its representation of the interactions that offer multiple pathways and multiple dimensions of those interactional pathways. This theory offers innumerable access points to begin the processes of resilience maintenance and enhancement.

The research itself is a strength; as noted, volunteer firefighters have not been well represented in the literature nor do they receive the level of support that other emergency services receive. The theory will contribute to the wellbeing of the very people who globally protect society's lives and property.

\section{Limitations}

The results of this work revealed a number of important and unique findings, but there are limitations to the study. Biological contributors to resilience were not explored in this research; the relationships between biology, environment, and resilience are exciting areas for further study. Generalisations are not possible with CGT which may be considered a limitation. Once the substantive theory was constructed with volunteer firefighters however it was explored 
with a convenience sample of five participants from outside the volunteer FRS (three career firefighters and two nurses). The external sample found the theory to be credible and original; it resonated with nurses and career firefighters, hence has high likelihood to be applied in other emergency services. As well, a limitation to the study may be in the health literay orientation of the participants; the theory may not resonate as deeply with FRS who lack a focus on mental health. Another limitation may be with the parameters of the data; although not simply a subjective experience, this theory was rendered through the lenses and experiences of the researcher and the firefighters. Overall, further exploration of the theory is required in other contexts, cultures, and countries.

\section{Conclusion}

This article overviewed a systematic inquiry into resilience in volunteer firefighters and discussed the substantive theory of resilience that emerged from the data.

This research intentionally explored firefighter perspectives of resilience in the underrepresented population of volunteer firefighters. The theory adds depth and breadth to the understanding and application of resilience in the volunteer sector internationally. It is of vital importance to understand resilience in all high-risk professions, and this CGT of resonates with career firefighters and nurses, and shows promise for utility across emergency services internationally. Importantly, the theory of resilience has relevance to occupational health in a wide variety of work settings globally by ensuring that policies and practices are grounded in evidence yet able to bridge the theory-to-practice gaps. Key take-away messages from this research include: resilience is multi-faceted and complex with six core categories that inter-relate amongst and between each other; resilience can be taught and strengthened by accessing one or 
more of the categories and sub-categories. Future research will test the theory in fire, nursing, and other emergency and global contexts.

\section{References}

Alexander, L. (2015). When the bells go down: Resilience and vulnerability in firefighters. PhD dissertation. University of Hertfordshire. Retrieved from: http://uhra.herts.ac.uk/handle/2299/17096?show=full

Alvarez, G. (2013). Gallows humor as a resiliency factor among urban firefighters with specific implications on prevalence rates of PTSD. Doctoral dissertation. Retrieved from ProQuest Dissertations and Theses. (Accession Order No. 3552414).

Antonovsky, A. (1996). The salutogenic model as a theory to guide health promotion. Health Promotion International, 11, 11-18.

Beaton, R., Murphy, S., Johnson, C., Pike, K., \& Corneil, W. (1998). Exposure to duty-related stressors in urban firefighters and paramedics. Journal of Traumatic Stress, 11(4), 821-828.

Benner, P., Sutphen, M., Leonard, V., \& Day, L. (2010). Educating nurses: A call for radical transformation. San Francisco, CA: Jossey-Bass.

Biron, C. \& Karanika-Murray, M. (2014). Process evaluation for organizational stress and well-being interventions: Implications for theory, method, and practice. International Journal of Stress Management, 21(1), 85-111.

Blaney, L. (2017). Working where the devil dances: A constructivist grounded theory of resilience in volunteer firefighters. Doctoral dissertation. Nottingham Trent University, Nottingham, UK. Available from: http://irep.ntu.ac.uk/id/eprint/31214/ 
Blaney, L. \& Brunsden, V. (2015). Resilience and health promotion in high risk professions: A pilot study of firefighters in Canada and the United Kingdom. The International Journal of Interdisciplinary Organizational Studies 10(2), 23-32.

Bonanno, G. A. (2004). Loss, trauma, and human resilience: Have we underestimated the human capacity to thrive after extremely aversive events? American Psychologist, 59(1), 20-28.

Bonanno, G., Brewin, C. R., Kaniasty, K., \& La Greca, A. (2010). Weighing the costs of disaster: Consequences, risks, and resilience in individuals, families, and communities. Psychological Science in the Public Interest, 11(1), 1-49.

Bonanno, G. \& Dimich, E. (2013). Annual Research Review: Positive adjustment to adversity trajectories of minimal impact resilience and emergent resilience. Journal of Child Psychology and Psychiatry, 54(5), 378-421.

Bonanno, G., Pat-Horenczyk, R., \& Noll, J. (2011). Coping flexibility and trauma: The perceived ability to cope with trauma (PACT) Scale. Psychological Trauma: Theory, Research, Practice, and Policy, (3)2, 117-129.

Bonanno, G., Romero, S., \& Klein, S. (2015). The temporal elements of psychological resilience: An integrative framework for the study of individuals, families, and communities. Psychological Inquiry, 26(2), 139-169.

British Columbia Professional Firefighters Association. (1999). Heart to Heart: A report on the Hazards Facing Fire Fighters. Burnaby: International Association of Fire fighters \& Canadian Labour Congress.

Bryant, R. A., \& Harvey, A. G. (1995). Predictors of distress. Journal of Nervous and Mental Diseases, 183, 267-271. 
Charmaz, K. (2014). Constructing grounded theory. $2^{\text {nd }}$ Edition. London: Sage Publications Ltd.

Childs, E. \& de Wit, H. (2014). Regular exercise is associated with emotional resilience to acute stress in healthy adults. Frontiers in Physiology, 5, Article 161, 1-7.

Chopko, B. \& Schwartz, R. (2009). The relation between mindfulness and posttraumatic growth: A study of first responders to trauma-inducing incidents. Journal of Mental Health Counselling, (31)4, 363-376.

Cote, M. \& Nightingale, A. (2012). Resilience thinking meets social theory: Situating social change in socio-ecological systems. Progress in Human Geography, 36(4), 475-489.

de Terte, I., Stephens, C., \& Huddleston, L. (2014). The development of a 3-part model of psychological resilience. Stress and Health, 30, 416-424.

del Ben, K., Scotti, J., Chen, Y., Fortson, B. (2006). Prevalence of posttraumatic stress disorder symptoms in firefighters. Work \& Stress, 20(1), 37-48.

Drucker, P. (2001). The essential Drucker. New York, NY: HarperCollins Publishers.

Ellis, B. (2015). Beyond risk and protective factors: Rethinking the role of stress in regulating child development and resilience. Keynote address given at Pathways to Resilience: Beyond Nature vs. Nurture conference. Halifax, Nova Scotia. June 17, 2015.

Fletcher, D. \& Sarkar, M. (2012). A grounded theory of psychological resilience in Olympic champions. Journal of Sport and Exercise, 13, 669-678.

Halpern, J., Gurevich, M., Schwartz, B., \& Brazeau, P. (2009). Interventions for critical incident stress in emergency medical services: A qualitative study. Stress \& Health: Journal of the International Society for the Investigation of Stress, 25(2), 139-149. 
Haslam, C., \& Mallon, K. (2003). A preliminary investigation of posttraumatic stress symptoms among firefighters. Work and Stress, 17, 277-285.

Haynes, H. \& Molis, J. (2015). US Firefighter Injuries 2014. NFPA No, FF110. Report. Quincey, MA: National Fire Protection Association.

Hill, R. (2015). Occupational related consequences for relatives of firefighters. $\mathrm{PhD}$ dissertation. Nottingham Trent University. Retrieved from Irep http://irep.ntu.ac.uk/id/eprint/28278/ .

Ho, S. \& Lo, R. (2012). Dispositional hope as a protective factor among medical emergency professionals: A preliminary investigation. Traumatology, 17, 4: 3-9.

Hobfoll, S. (2014). Resource caravans and resource caravan pathways: A new paradigm for trauma responding, Intervention, 12, Suppl 1, 21-32.

Jeannette, J. M., \& Scoboria, A. (2008). Firefighter preferences regarding post-incident intervention. Work \& Stress, 22(4), 314-326.

Johnson, J. (2010). Understanding differences in stress and coping between full-time and paidon-call firefighters. PhD dissertation. Accessed from Proquest. UMI 3428172.

Karter, M. Jr. \& Stein, G. (2013). US Fire Department Profile. Report prepared for the National Fire Protection Association, Quincy, MA. Retrieved August 21, 2014 from: http://www.nfpa.org/research/reports-and-statistics/the-fire-service/administration/us-

\section{fire-department-profile}

Mancini, A. \& Bonanno, G. (2009). Predictors and parameters to resilience: Towards an individual differences model. Journal of Personality, 77(6), 1805-1831. 
Masten, A. (2015, June). Resilience in human development: Interdependent adaptive systems in theory and action. Keynote address given at the Pathways to Resilience III conference. Halifax, NS.

Masten, A. (2001). Ordinary magic: Resilience processes in development. American Psychologist, 56(3), 227-238.

Masten, A. \& Narayan, J. (2012). Child development in the context of disaster, war, and terrorism: Pathways of risk and resilience. Annual Review of Psychology, 63, 227-257.

Mayan, M. (2009). Essentials of Qualitative Inquiry. Walnut Creek, CA: Left Coast Press.

McAllister, M. \& McKinnon, J. (2009). The importance of teaching and learning resilience in the health disciplines: A critical review. Nurse Education Today, 29, 371-379.

McMahon, C. (2010). A Phenomenological Study of Stress and Coping in the Fire Service. (Doctoral dissertation). Retrieved from ProQuest Dissertations and Theses. (UMI number: 3410314)

Meffert, U. (2015.) Gear Ball. [Rotating puzzle]. Meffert's Puzzles and Games. http://www.mefferts.com/

Mental Health Commission of Canada. (2013). National Standard of Canada for Psychological Health and Safety in the Workplace. Available from: http://www.mentalhealthcommission.ca/English/national-standard

National Fire Protection Association (NFPA). (2016). Number for firefighters in Canada: 20132015. NFPA Analysis and Research. Quincey, MA: Author. Retrieved from: http://www.nfpa.org/News-and-Research/Fire-statistics-and-reports/Fire-statistics/Thefire-service/Administration/Fire-departments-in-Canada

Noblet, A., \& LaMontagne, A. (2006). The role of workplace health promotion in addressing 
job stress. Health Promotion International, 21(4), 346-353.

Panter-Brick, C. (2014). Health, risk, and resilience: Interdisciplinary concepts and applications. Annual Review of Psychology, 43: 431-448.

Regehr, C. (2009). Social support as a mediator of psychological distress in firefighters. Irish Journal of Psychology, (30)1-2, 87-98.

Regehr, C., Dimitropoulos, G., Bright, E., George, S., \& Henderson, J. (2005). Behind the brotherhood: Rewards and challenges for wives of firefighters. Family Relations, 5(3), 423-435.

Richardson, G. (2002). The metatheory of resilience and resiliency. Journal of Clinical Psychology, 58(3), 307-321.

Shakespeare-Finch, J. (2011). How emergency service workers cope with, and grow from, work-related stress and trauma. In: Wayfinding through Life's Challenges. K. Gow \& M. Celinski (Eds). Hauppauge, NY: Nova Science Publishers Inc.

Sliter, M., Kale, A., \& Yuan, Z. (2013). Is humor the best medicine? The buffering effect of coping humor on traumatic stressors in firefighters. Journal of Organizational Behavior, $35,257-272$.

Southwick, S., Bonanno, G., Masten, A., Panter-Brick, C., \& Yehuda, R. (2014). Resilience definitions, theory, and challenges: Interdisciplinary perspectives. European Journal of

Psychotraumatology, 5. 10.3402/ejpt V5 25338. http://doit.org/a0.3402/ejptv5.25338

Southwick, S., Litz, b., Charney, D., \& Friedman, M. (Eds). (2011). Resilience and mental health: Challenges across the lifespan. (pp. xi-xv). Cambridge, UK: Cambridge University Press. 
Tehrani, N. (2011). Building resilient organizations in a complex world. In: N. Tehrani (Ed.) Managing trauma in the workplace. New York, NY: Routledge.

Uhl-Bien, M. \& Marion, R. (Eds.) (2008). Complexity Leadership part 1: Conceptual Foundations. Charlotte, NC: IAP, Information Age Publishing.

Ungar, M. (2011). The social ecology of resilience: Addressing contextual and cultural ambiguity of a nascent construct. American Journal of Orthopsychiatry, 81(1), 1-17.

Vann-Ward, T., Morse, J., \& Charmaz, K. (2017). Preserving self: Theorizing the social and psychological processes of living with Parkinson disease. Qualitative Health Research, 27(7), 964-982.

Von Bertalanffy, L. (1969). General Systems Theory, Foundations, Development, Applications. New York: G. Braziller.

Wagner, S., McFee, J., \& Martin, C. (2010). Mental health implications of fire service membership. Traumatology, 16(2), 26-32.

Wagnild, G., \& Young, H. (1993). Development and psychometric evaluation of the resilience scale. Journal of Nursing Scholarship, 1(2), 165-178.

Youssef, C. \& Luthans, F. (2007). Positive organizational behavior in the workplace: The impact of hope, optimism, and resilience. Journal of Management, 33(5), 774-800. 


\section{Figure 1: Resilience in volunteer firefighters}

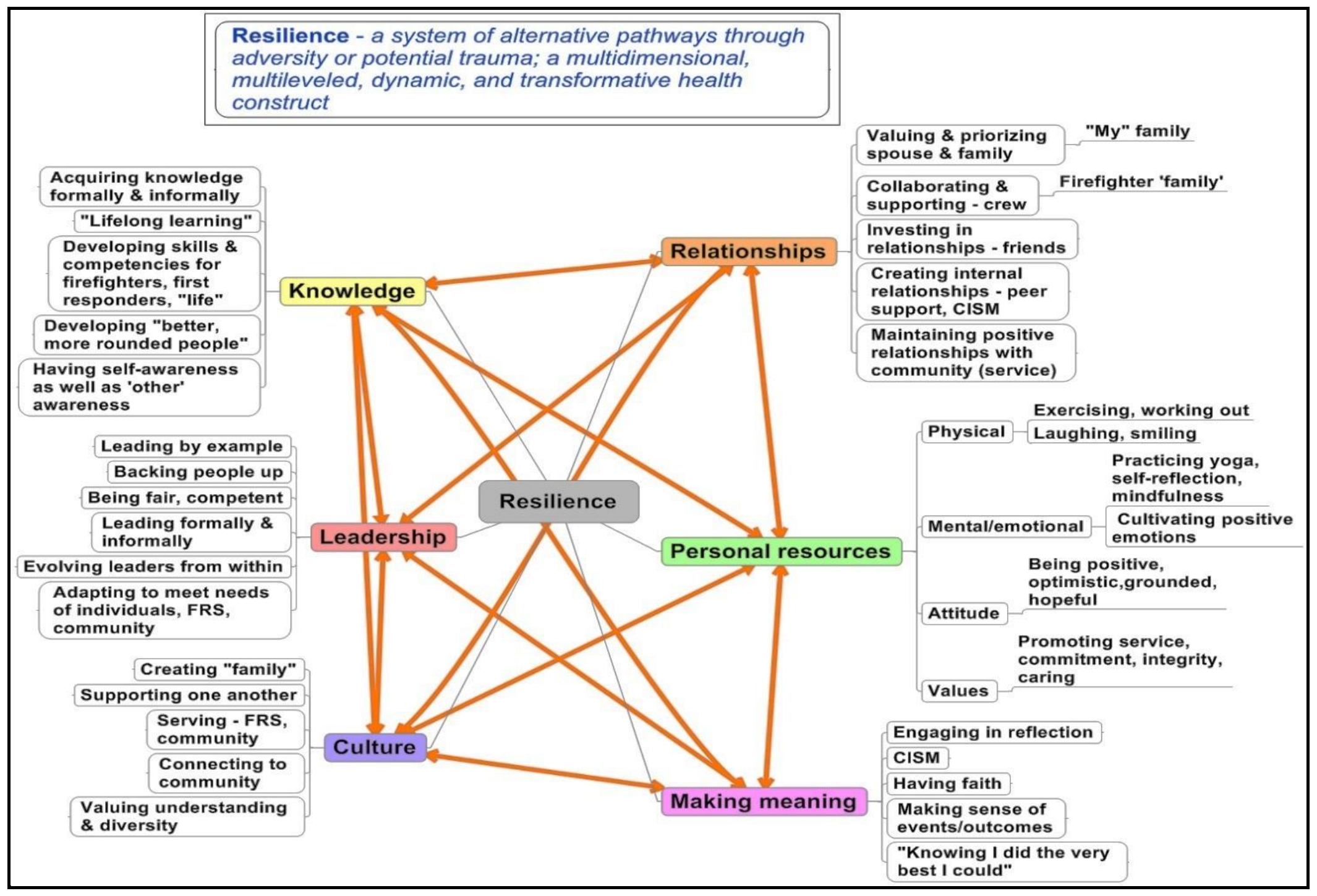

